\title{
Conductas conflictivas y convivencia escolar: análisis desde el modelo ecológico
}

\section{Conflictive behaviors and school convivencia: Analysis from the ecological model}

\author{
Azucena de la Concepción Ochoa CERVANTES* \\ PAMELA GARBUS ${ }^{* *}$ \\ ANDREA Morales OSORNIO ${ }^{* * *}$
}

El objetivo de este trabajo es analizar la prevalencia de percepción de tres conductas conflictivas: desinterés, desmotivación y deshonestidad académica en estudiantes de secundarias públicas de un municipio del semidesierto queretano de México. El estudio en el que se basa es de corte epidemiológico observacional, analítico transversal, y el muestreo es probabilístico y estratificado según el tipo de escuela y nivel de marginación de las secundarias. La convivencia escolar se aborda desde una perspectiva ecológica que establece dos dimensiones para su estudio: la personal, que incluye la edad y el sexo, y la institucional, integrada por tres subsistemas: macrosocial, mesosocial y microsocial. Se encontró una dependencia entre la percepción de conductas conflictivas y el nivel de marginación de la escuela: las escuelas con niveles menores de marginación reportaron mayor prevalencia de percepción de conductas conflictivas, y también se observó una mayor prevalencia en las escuelas generales en comparación con las telesecundarias. El estudio plantea líneas de indagación sobre las condiciones macrosociales de la institución y su vínculo con la convivencia escolar.

\section{Palabras clave:}

convivencia escolar, modelo ecológico, conductas conflictivas, escuelas secundarias

Recibido: 2 de enero de 2021 | Aceptado para su publicación: 9 de agosto de 2021 |

Publicado: 22 de septiembre de 2021

Cómo citar: Ochoa Cervantes, A. C., Garbus, P. y Morales Osornio, A. (2021). Conductas conflictivas y convivencia escolar: análisis desde el modelo ecológico. Sinéctica, Revista Electrónica de Educación, (57), e1237. https://doi.org/10.31391/S2007-7033(2021)0057-012 
The aim was to analyze the prevalence of the perception of three conflictive behaviors: Disinterest, lack of motivation and academic dishonesty of public high school students of a municipality in the semi-desert area of Queretaro. We carried out an observational epidemiological, cross-sectional analytical study. The sampling was probabilistic and stratified according to the type and marginalization level of the secondary schools. We proposed a Systemic Ecological perspective for the analysis of school convivencia which establishes two dimensions for its study, the personal and the institutional, which integrates three subsystems: Macro-social, meso-social, and micro-social. It was found that there is a dependence between the perception of conflictive behaviors and the level of marginalization of the school, schools with lower levels of marginalization have a higher prevalence of perception of conflictive behaviors. There is a higher prevalence of perception of conflictive behaviors in general schools than in telesecundarias. The study proposes lines of research related with the macro-social conditions of the institution and their link with school convivencia.

\section{Keywords:}

school convivencia, ecological model, conflictive behaviors, high school

\footnotetext{
* Doctora en Psicología y Educación por la Universidad Autónoma de Querétaro. Docente de la Facultad de Psicología de la Universidad Autónoma de Querétaro. Responsable del Observatorio de la Convivencia Escolar de la misma universidad e integrante del comité directivo de la Red Latinoamericana de Convivencia Escolar y de la Red Mexicana de Aprendizaje Servicio. Cuenta con el reconocimiento de investigadora nacional nivel 1 por el Conacyt. Líneas de investigación: Convivencia escolar, participación de niños y niñas, educación inclusiva. Correo electrónico: azus@uaq.mx/ https:// orcid.org/0000-0003-4515-9069

** Doctora en Salud Mental Comunitaria por la Universidad Nacional de Lanús, Argentina. Docente e investigadora y coordinadora del Observatorio y la maestría en Salud Mental de la Infancia y la Adolescencia de la Facultad de Psicología de la Universidad Autónoma de Querétaro. Reconocimiento de investigadora nacional nivel 1 por el Conacyt. Líneas de investigación: procesos y prácticas de atención en salud y salud mental infantojuvenil, estudios epidemiológicos en salud mental, formación docente para la inclusión educativa, derechos y ciudadanía en adolescentes. Correo electrónico: pgarbus@gmail.com/https://orcid.org/0000-0003-4286-1362

*** Maestra en Salud Mental de la Infancia y la Adolescencia por la Universidad Autónoma de Querétaro. Docente de escuela secundaria y preparatoria. Correo electrónico: amorales38@alumnos.uaq. mx/ https://orcid.org/0000-0001-8497-9989
} 


\section{INTRODUCCIÓN}

n las últimas dos décadas en México, la convivencia escolar ha sido abordada

4 desde múltiples perspectivas y enfoques; sin embargo, se trata de una temática compleja, inacabada y que aún merece ser indagada por la investigación educativa. Desde su aparición como tema emergente en la investigación educativa, la convivencia escolar se ha visto asociada al estudio de algunas variables como la violencia, la disciplina, las habilidades sociales, que vinculan la convivencia a factores individuales (Askell-Williams, Cefai \& Fabri, 2013; Chen \& Wei, 2011; Crespo-Ramos et al., 2017; Flaspohler et al., 2009; Trianes et al., 2009; Varela, Ávila y Martínez, 2013); en la mayoría de los casos, el abordaje de estos conflictos es descontextualizado del contexto donde emergen. Muy pocos estudios dan cuenta de la relación entre la conflictividad y variables contextuales como nivel socioeconómico o tipo de escuela (Ascorra et al., 2016; Aguilera, Muñoz y Orozco, 2007) o estudios que atribuyen un clima escolar "violento" a una condición, a "contextos de alta vulnerabilidad social" (Tijmes, 2012). Gaeta et al. (2020) sugieren un abordaje sistémico para explicar los problemas de convivencia que emergen en el espacio escolar.

Definimos la convivencia como la relación interpersonal que se establece entre los miembros de la comunidad escolar, la cual es mediada por la institución e influye en la forma de interactuar entre las personas. Siguiendo a Bronfenbrenner (1987), en este trabajo proponemos para el análisis de la convivencia escolar una perspectiva ecológica que sostiene que el desarrollo de las personas debe estudiarse de manera situada en un contexto, es decir, tomando como objeto de estudio las relaciones entre las personas y los entornos.

Para Bronfenbrenner (1987),

... el desarrollo humano comprende el estudio científico de la progresiva acomodación mutua entre un ser humano activo, en desarrollo y las propiedades cambiantes de los entornos inmediatos en los que vive la persona en desarrollo, en cuanto este proceso se ve afectado por las relaciones que se establecen entre estos entornos, y por los contextos más grandes en los que están incluidos los entornos (p. 40).

El autor plantea la descripción del contexto a partir de lo que denomina ambiente ecológico, que define como "un conjunto de estructuras seriadas, cada una de las cuales cabe dentro de la siguiente..." (Bronfenbrenner, 1987, p. 23). De este modo se refiere al micro-, meso- y macrosistema.

Retomando estas ideas, para analizar la convivencia, presentamos dos dimensiones para su estudio: la personal, que incluye aspectos como la edad y el sexo, y la institucional, integrada, a su vez, por tres subsistemas: macrosocial (contexto), mesosocial (institución) y microsocial (aula) (ver figura 1). 


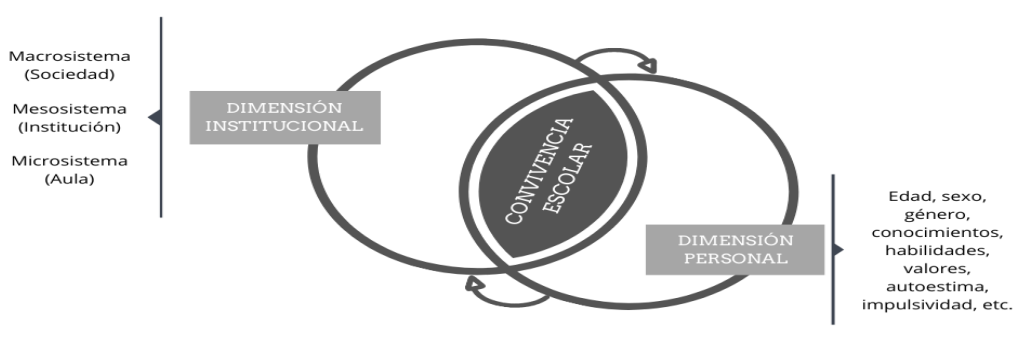

Figura 1. Modelo ecológico de la convivencia escolar.

\section{Microsistema (aula)}

Refiere el entorno inmediato de los alumnos. El aula se considera un escenario concreto que comprende un entorno físico y de interrelaciones que se dan entre las personas, en el cual interactúan los estudiantes, los profesores, los materiales educativos, el espacio físico, y las normas del aula explícitas e implícitas.

\section{Mesosistema (institución)}

Este amplía las interrelaciones de contextos y admite más de dos entornos interrelacionados en los que los estudiantes viven y participan. En este sentido, el mesosistema está conformado por actores-roles y vínculos (director, maestros, alumnos, personal administrativos, padres de familia), formas de comunicación y por la organización (dirección y gestión, coordinación pedagógica, participación, normas y resolución de conflictos).

\section{Macrosistema (sociedad)}

Concierne a los diferentes entornos, locales, estatales y nacionales, que tienen impacto en la convivencia en la escuela, y en los que el estudiante no participa directamente, pero sí recibe su influencia. Aquí encontramos los factores tecnológicos y de comunicación, religiosos, sociales y familiares, factores culturales, factores políticos, factores económicos y laborales.

Así, la convivencia se desarrolla como resultado de la participación del individuo en estos sistemas, en los que desempeña roles, realiza actividades y mantiene relaciones interpersonales. Sin embargo, es preciso reconocer que esta interacción, en ocasiones, se ve afectada, entre otros aspectos, por conductas conflictivas que pueden influir negativamente en la convivencia escolar, dado que interfieren con el desarrollo del individuo. Si bien estas conductas conflictivas son mostradas por los sujetos en las distintas interacciones cotidianas, son también mediadas por la institución de tal modo que las formas de abordarse pueden propiciarlas, o bien, prevenirlas.

En el contexto escolar no todas estas conductas son igualmente visibles ni todas causan la misma preocupación. Esto dificulta su reconocimiento y, por ende, la posibilidad de evitar conflictos que pueden afectar la convivencia escolar. La figura 2 presenta tres conductas conflictivas que consideramos pueden alterar la convivencia escolar y que son objeto de estudio en este artículo. 


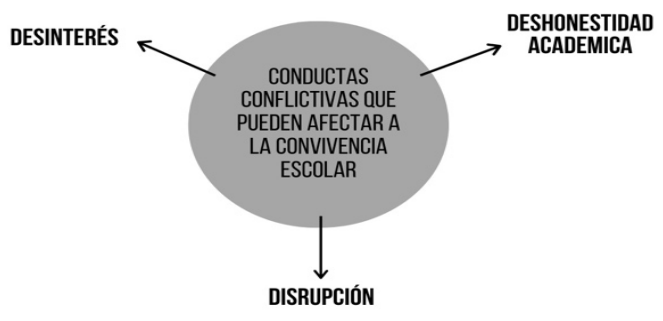

Figura 2. Conductas conflictivas que pueden afectar la convivencia escolar.

En este trabajo nos centramos en la percepción de tres conductas que tienden a propiciar conflictos y pueden afectar la convivencia escolar: la deshonestidad académica, el desinterés y la disrupción. La percepción de estas conductas forma parte de lo que se denomina clima de convivencia y, de acuerdo con la postura ecológica, "lo que cuenta para la conducta y el desarrollo es el ambiente como se lo percibe, más que como pueda existir en la realidad objetiva" (Bonfrenbrenner, 1987, p. 24).

Una conducta es conflictiva cuando emerge y a ella le sigue un abordaje inadecuado por parte de la institución, es decir, la conducta en sí misma no provoca el conflicto; este proviene de lo que sucede en las interacciones dentro de ese contexto. Entonces, siguiendo el modelo ecológico de la convivencia escolar, para el análisis de estas conductas conflictivas que afectan dicha convivencia deben considerarse tanto las características de la institución como las variables del contexto social donde se desarrollan la convivencia y los conflictos que la afectan. El objetivo de este trabajo es analizar la prevalencia de estas tres conductas conflictivas percibidas por el estudiantado y establecer la relación con algunas variables del mesosistema (tipo de escuela) y del macrosistema (nivel de marginación) en estudiantes de escuelas secundarias públicas de un municipio del semidesierto queretano.

Desde el modelo ecológico, consideramos que los entornos influyen en las personas que ahí se encuentran; en particular, el macrosistema, según Bronfenbrenner, es el entorno global que explica la homogeneidad y las correspondencias entre los demás subsistemas. Para el autor, este es el más estable y su influencia sobre los demás es crítica. En este artículo planteamos un enfoque que recupera estas conductas (deshonestidad académica, desmotivación y disrupción), las cuales, a excepción de la disrupción, no han sido abordadas como una conducta que puede alterar la convivencia escolar. En este sentido, el propósito final de este trabajo es sumar al entendimiento del fenómeno de la convivencia escolar al incorporar para su estudio estas tres conductas conflictivas y, en especial, la percepción que los propios estudiantes tienen de ellas.

\section{ESTUDIOS SOBRE LOS CONFLICTOS QUE AFECTAN LA CONVIVENCIA}

La convivencia escolar es un fenómeno complejo que involucra diversas variables, dimensiones y sujetos. Cada uno aporta al entendimiento de cómo se construyen, o no, relaciones interpersonales positivas -que permiten establecer condiciones para el aprendizaje y la inclusión- en el contexto escolar. En este artículo abordamos la percepción por parte del estudiantado de tres conductas conflictivas que suelen 
afectar la convivencia escolar y las relacionamos con variables de la dimensión institucional: tipo de escuela (mesosistema) y nivel de marginación (macrosistema), así como con el sexo, que es una variable de la dimensión personal.

Como ya mencionamos, nos centraremos en tres de las conductas conflictivas que no motivan tanta preocupación en la investigación educativa, situación que se evidencia por la falta de estudios que indagan sobre estas para el entendimiento de la convivencia escolar. Consideramos que son relevantes para el estudio de la convivencia debido a que su abordaje inadecuado, ya sea por indiferencia o por las acciones que pone en marcha la institución ante su incidencia, transmite valores que resultan contrarios a los fines educativos, como el fomentar una convivencia positiva, es decir, que permita la construcción de un ambiente inclusivo.

A continuación, abordamos los antecedentes generales de la deshonestidad académica, la desmotivación y la disrupción.

Desde 2012, algunos estudios demostraron el impacto de la deshonestidad académica en la convivencia escolar y en la formación del alumnado de educación básica (Ochoa y Diez-Martínez, 2013; Ochoa y Diez-Martínez, 2012a). La escuela es un espacio privilegiado para el aprendizaje, a través del ejemplo y las prácticas cotidianas que se llevan cabo, de valores y pautas sociales. Entendemos al aprendizaje como "un cambio relativamente permanente en la conducta o en su potencialidad que se produce a partir de la experiencia” (Hergenhahn, 1976, citado en Castro, 2019); es un proceso constante en el que intervienen tanto aspectos internos del propio estudiante como del nivel y tipo de interacción que este establece con el medio.

Ninguna educación es aséptica, es decir, implícita o explícitamente siempre transmite valores. La acciones o comportamientos que atentan contra la legalidad en algunas escuelas, como desigualdad en el proceso de selección, injusticia y falta de transparencia en la acreditación, incoherencia en la gestión de insumos y materiales, la inconsistencia o impunidad en la aplicación de las reglas, el hecho de copiar en los exámenes, copiar o comprar las tareas, falsificar firmas, por parte de los alumnos, son actos de deshonestidad académica que impactan en el bienestar de la colectividad, ya que afectan la confianza y, por ende, las relaciones entre los miembros de la comunidad y pueden ser un generador de problemas de convivencia al interior de los centros escolares.

Es necesario considerar este conflicto como parte de la convivencia y la formación ciudadana, debido a que se pone en juego información que permite entender las normas y su aplicación justa o discrecional. No obstante, son escasos los estudios que se han desarrollado sobre este tema en la población infantil y adolescente (Eisenberg, 2004; Morey-López et al., 2013; Murdock, Hale \& Weber, 2001; Nora \& Zhang, 2010; Sureda, Comasy, Oliver, 2015; Reskala, 2020).

Por su parte, la desmotivación puede desencadenar conductas conflictivas en el alumnado y ser una fuente de conflicto; en consecuencia, puede alterar la convivencia en las aulas y las escuelas. La desmotivación del alumnado puede ser causada por una práctica docente tradicional que no promueva la participación y origine el círculo de desmotivación (Ortega y Del Rey, 2006). En 2013, Ochoa, Salinas y Diez-Martínez mostraron que el $97 \%$ del alumnado de secundaria que participó en su estudio percibía aburrimiento y desinterés en las actividades escolares. Esta falta de interés suele 
afectar la relación estudiante-docente y también las relaciones entre el estudiantado al interior del aula. Se espera que las actividades educativas, a la par de ser fuente de aprendizajes escolares, sean promotoras de mejores relaciones entre los miembros de una comunidad que comparten, durante un espacio y tiempo, un objetivo común, de ahí que la motivación sea un elemento esencial de las relaciones y la convivencia.

A su vez, la desmotivación puede generar la emergencia de otros comportamientos que deterioran las relaciones en las aulas y en la escuela. Uno de ellos es la disrupción, la cual podemos definir como todo comportamiento que tiende a dificultar la enseñanza de tal modo que afecta al grupo al alterar la dinámica en el proceso de enseñanza y aprendizaje; por lo general, se manifiesta con ruidos o movimientos molestos, interrupciones al docente o al alumnado. La disrupción es una conducta conflictiva que deteriora, principalmente, la relación docente-estudiante, pues, en muchos casos, este tipo de comportamientos se toma como una falta a la persona, lo que causa malestar y malas relaciones.

Aunado a lo anterior, si la disrupción no se atiende, puede escalar a situaciones graves que provoquen violencia, abandono o fracaso escolar. Este comportamiento es reconocido como de alta prevalencia en la secundaria (Gordillo, Rivera-Calcina y Gamero, 2014; Ochoa y Diez-Martínez, 2012b; Ochoa, Salinas y Diez-Martínez, 2013; Jurado y Tejeda, 2019; Jurado, Lafuente y Justiniano, 2020; Martínez et al., 2020).

Por otra parte, algunos estudios indagan cuestiones relacionadas tanto con el nivel microsocial como con el mesosocial, ambos de la dimensión de la institución. Torres-Rivera y Florencio (2019) analizan las estrategias didácticas que emplean los docentes, el modelo educativo de la institución y la función de la educación superior en la formación ciudadana de estudiantes. Los resultados de su estudio sugieren que el diálogo, la confianza y el aprecio por la diversidad son las prácticas que los docentes identifican como el saber convivir. Cabe señalar que las intervenciones descritas por estos autores se realizaron a nivel institucional.

Grau y García (2017) documentan una intervención en la cual aplicaron estrategias basadas en el diálogo y la participación que reforzaron el vínculo de los estudiantes con la escuela. Se organizaron talleres artísticos que abordaron los derechos de la infancia, juegos dirigidos por el alumnado para establecer nuevos vínculos con otros compañeros y asambleas semanales de alumnos, profesores y familiares. Algunos de los resultados fueron una mayor participación de las familias y una disminución en los enfrentamientos, peleas, discusiones, insultos, exclusión del grupo y desmotivación.

Otro aspecto estudiado como parte del mesosistema es la participación y su importancia en la construcción de climas favorables para la convivencia y el aprendizaje. Al respecto, un estudio cualitativo de Ascorra, López y Urbina (2016) reporta que, en Chile, en las escuelas que consideraron para su estudio, la participación de los estudiantes resultaba tutelada. Por su parte, Ochoa (2019) muestra en una investigación en México que la participación promovida en las escuelas es dirigida por los adultos. En la misma línea, Ochoa y Pérez (2019) demuestran que la aplicación de la metodología aprendizaje servicio en escuelas secundarias ofrece una mejora del clima escolar, la modificación de prácticas pedagógicas y el posicionamiento del alumnado como protagonista. 
Asumir la postura ecológica nos permite entender la emergencia de los conflictos no solo como producto del individuo, sino como condicionados a variables que caracterizan el contexto de la institución donde estos conflictos emergen.

\section{EL CONTEXTO DE LA EDUCACión SECUNDARIA EN MÉXICo}

El nivel de secundaria es el tercer tramo de la educación obligatoria en México. Asisten estudiantes de entre 12 y 15 años en promedio. Los tipos de servicio que se ofrecen son general, técnica, telesecundaria, comunitaria y para trabajadores. La oferta de servicios en cada uno de los niveles de la educación obligatoria que brinda el sistema educativo nacional es la respuesta que el Estado ha dado a la población para que, sin importar el grado de marginación y el tamaño de la localidad donde habita, esta pueda ejercer su derecho a acceder a la educación. No obstante, esto no garantiza la calidad de la educación brindada ni asegura tampoco el acceso de toda la población (INEE, 2018).

De acuerdo con los datos del Instituto Nacional de Evaluación de la Educación (INEE, 2018), el servicio general concentra la mitad de la matrícula y de la plantilla docente y a una de cada tres escuelas. Por su parte, las telesecundarias son servicios educativos que siguen un modelo de enseñanza que combina la educación a distancia con la educación presencial, y se ubican sobre todo en zonas rurales o de difícil acceso o con baja concentración demográfica (Coordinación General @prende.mx, 2020); estas son las que tienen mayor presencia en este nivel educativo: $47.8 \%$, aunque su matrícula abarca el $21.4 \%$ de los estudiantes y su plantilla docente representa el $17.9 \%$. Vale la pena considerar que en ellas hay un docente por grado y no uno por asignatura como sucede en el tipo de servicio general. Las secundarias técnicas, por otro lado, concentran el $27.1 \%$ de la matrícula, el $24.7 \%$ de los docentes y apenas el $12 \%$ de las escuelas.

Del análisis de reportes oficiales, inferimos que no hay uniformidad en la distribución de los servicios educativos en el territorio nacional. Los servicios general y técnico en secundaria se ubican, principalmente, en localidades urbanas con bajo grado de marginación, mientras que las escuelas indígenas, comunitarias y telesecundarias son las que atienden, en su mayoría, a la población en localidades rurales y con alto grado de marginación. Estas se encuentran en localidades de menos de 2,500 habitantes, en donde siete de cada diez alumnos están matriculados en ellas. Vale considerar que nueve de cada diez telesecundarias se sitúan en poblados de media, alta y muy alta marginación (INEE, 2018).

Para el ciclo 2016-2017, el 55.9\% de escuelas y planteles se encontraban en localidades con alto y muy alto grado de marginación; en las de alta y muy alta marginación para el nivel secundaria, el servicio con el mayor número de escuelas era el de telesecundaria, con 14,376 centros escolares. Respecto a las secundarias generales, 6,524 se situaban en localidades de medio y bajo nivel de marginación (53.4\%); en los lugares de muy bajo nivel de marginación, este servicio también prevalecía, con 2,923 escuelas (77.2\%) (INEE, 2018).

A partir de los datos presentados, el INEE (2018) afirma que estos estudiantes tienen condiciones poco favorables para poder asistir a la escuela, ya que, en la mayoría 
de los casos, las zonas de alta marginación se enfrentan a ciertas carencias que se reflejan en la falta de recursos de las familias, el nivel de pobreza, el deterioro de las escuelas o la lejanía de estas, los riesgos naturales y humanos que genera el traslado a los centros escolares, así como la escasez de servicios y modalidades educativas.

Con los datos presentados, podemos afirmar que el tipo de escuela o el grado de marginación de estas -que la Secretaría de Educación Pública retoma del Consejo Nacional de Población (Conapo) para caracterizar a una escuela según los indicadores socioeconómicos de la localidad donde se encuentra- son variables que deben ser consideradas para comprender lo que acontece en la escuela, específicamente lo relacionado con la presencia de conflictos de convivencia.

\section{MÉTODo}

Nuestro estudio fue de corte epidemiológico observacional, analítico transversal (Villa et al., 2012). La población objetivo la formaron 3,762 estudiantes de secundarias públicas del ciclo escolar 2018-2019 de un municipio del semidesierto queretano. El tipo de muestra fue probabilística, estratificada según el tipo de escuela, telesecundaria o general (los dos tipos de servicios educativos públicos que se ofrecen en este nivel en el municipio donde se llevó a cabo el estudio) y nivel de marginación de las secundarias y por racimos, que fueron los grupos escolares. La estrategia utilizada para aleatorizar fue tómbola; en la primera etapa se sortearon las escuelas dependiendo del tipo de escuela y nivel de marginación, y en la segunda, se hizo un sorteo de los grupos a los que se les enviaría carta de consentimiento informado para la aplicación del cuestionario; este sorteo se realizó junto con los directores de cada escuela.

El nivel de marginación se refiere a un índice multidimensional que mide en las entidades federativas y los municipios su intensidad por medio de nueve formas de exclusión agrupadas en cuatro dimensiones: educación, vivienda, distribución de la población e ingresos monetarios. La dimensión educación está formada por indicadores de analfabetismo y de no conclusión de la educación primaria. La de vivienda, por indicadores sobre la inexistencia de servicios: drenaje, excusado, energía eléctrica, agua entubada, además de los indicadores de piso de tierra y hacinamiento. La de distribución de la población, por el porcentaje de población residente en localidades con menos de 5,000 habitantes, y la de ingresos, por la población con ingresos de hasta dos salarios mínimos (Conapo, 2016).

El cálculo de la muestra se realizó con un margen de error del 3.5\% y un nivel de confianza del 95\%; esto dio como resultado una muestra representativa de 649 estudiantes. A cada uno de los participantes se les solicitó el asentimiento, y el consentimiento informado fue firmado por sus padres o tutores. Cabe mencionar que el estudio en el cual se enmarca este trabajo fue aprobado por el comité de ética de la investigación de la Dirección de Investigación y Posgrado de la Universidad Autónoma de Querétaro.

El instrumento que se utilizó fue una adaptación de la Escala para la Valoración de Conflictos que afectan la Convivencia Escolar (ECACE), de Salinas y Ochoa (2016), que mide, entre otras variables, las analizadas en este trabajo. La adaptación fue piloteada y obtuvo un coeficiente de consistencia interna de alfa de Cronbach $a=0.93$. 
Así, la pertinencia de su elección estuvo dada por abordar diferentes variables de la convivencia escolar, por el nivel de coeficiente de consistencia interna y por ser un instrumento autoaplicable, lo que era imprescindible puesto que su aplicación fue de forma simultánea a cada grupo sorteado.

La ECACE consta de dos escalas, la de conductas conflictivas y la de conductas violentas. Para este trabajo, retomamos los datos de la escala de conductas conflictivas, ya que consideramos que la relación entre estas conductas y las variables indagadas tipo de escuela, nivel de marginación y sexo- ha sido poco abordada. Asimismo, como ya mencionamos, pretendemos sumar a la comprensión del fenómeno de la convivencia escolar incorporando variables poco utilizadas para su estudio (ver tabla 1).

Tabla 1. Indicadores de la escala de conductas conflictivas de la ECACE

\begin{tabular}{|c|c|c|c|}
\hline Escala & Definición conceptual & Subescalas & Indicadores \\
\hline \multirow{5}{*}{$\begin{array}{l}\text { Conductas } \\
\text { conflictivas }\end{array}$} & \multirow{5}{*}{$\begin{array}{l}\text { Comportamientos que } \\
\text { pueden provocar con- } \\
\text { flictos, pero no necesa- } \\
\text { riamente desencadenar } \\
\text { una situación que altera } \\
\text { la convivencia escolar o } \\
\text { violencia }\end{array}$} & $\begin{array}{l}\text { Deshonestidad acadé- } \\
\text { mica percibida en el } \\
\text { entorno }\end{array}$ & $\begin{array}{l}\text { Ser testigo de comportamientos que } \\
\text { atentan contra la legalidad dentro del } \\
\text { sistema escolar }\end{array}$ \\
\hline & & $\begin{array}{l}\text { Deshonestidad acadé- } \\
\text { mica vivida }\end{array}$ & $\begin{array}{l}\text { Ser víctima de comportamientos que } \\
\text { atentan contra la legalidad dentro del } \\
\text { sistema escolar }\end{array}$ \\
\hline & & $\begin{array}{l}\text { Deshonestidad acadé- } \\
\text { mica provocada }\end{array}$ & $\begin{array}{l}\text { Participar en comportamientos que aten- } \\
\text { tan contra la legalidad dentro del sistema } \\
\text { escolar }\end{array}$ \\
\hline & & Desmotivación & $\begin{array}{l}\text { Sentir desgano o desinterés en el proceso } \\
\text { de enseñanza y aprendizaje }\end{array}$ \\
\hline & & Disrupción & $\begin{array}{l}\text { Comportamientos que tienen la intención } \\
\text { de interrumpir el proceso de enseñanza } \\
\text { que generalmente crea tensión entre el } \\
\text { docente y el estudiante }\end{array}$ \\
\hline
\end{tabular}

\section{Análisis estadístico}

Para el análisis de datos, sumamos los puntajes de cada participante y obtuvimos su puntuación y, con base en esta, cuantificamos los promedios de cada sexo, nivel de marginación y tipo de escuela. Calculamos puntos de corte a partir de la media, más y menos una desviación estándar, para estimar los niveles de conflictividad.

Realizamos pruebas t de Student para muestras independientes a fin de encontrar diferencias por sexo; cuando la significancia fue menor de 0.05 , calculamos puntos de corte para cada sexo. Para valorar las diferencias de promedios por nivel de marginación, llevamos a cabo análisis de varianza ANOVA de un factor con los puntajes de los estudiantes para cada variable. Para determinar las diferencias de promedios por tipo de escuela, efectuamos pruebas t de Student para muestras independientes en las cuales los grupos fueron secundaria general y telesecundaria.

Para calcular la independencia estadística entre niveles de marginación y cada variable, recurrimos a la prueba Chi-cuadrada de Pearson y, del mismo modo, para el tipo de escuela. 


\section{RESULTADOS}

Prevalencia por niveles de percepción de conflictividad: escala general

La escala de conductas conflictivas se compone de cinco subescalas: deshonestidad académica percibida en el entorno, deshonestidad académica vivida, deshonestidad académica provocada, desmotivación y disrupción. Para el análisis de la percepción de conductas conflictivas, la escala general, se establecieron tres niveles: conflictividad baja, con base en el puntaje medio general menos una desviación estándar (ミ6.1), conflictividad media, entre 6.1 y 19.9, y conflictividad alta, puntaje promedio más una desviación estándar ( $\geqq 19.9$ ).

Entendemos por prevalencia la proporción de individuos de una población que presentan el evento en un momento determinado, en este caso, la percepción de cada una de las conductas conflictivas estudiadas; así, la prevalencia de percepción de conflictividad baja para la muestra total fue del 18.3\%, de conflictividad media, del $64.3 \%$ y de conflictividad alta, del 17.4\% (ver tabla 2).

Tabla 2. Prevalencias por nivel de conflictividad por nivel de marginación

\begin{tabular}{|c|c|c|c|c|c|}
\hline \multirow{2}{*}{$\begin{array}{c}\text { Nivel de } \\
\text { conflictividad }\end{array}$} & \multicolumn{4}{|c|}{ Nivel de marginación } & \multirow{2}{*}{ Total } \\
\cline { 2 - 5 } & Bajo & Medio & Alto & Muy alto & \\
\cline { 2 - 5 } & $\%$ & $\%$ & $\%$ & $\%$ & $\%$ \\
\hline Alta & 25.4 & 20 & 9.9 & 6.3 & 17.4 \\
\hline Media & 58.2 & 64.4 & 66.3 & 62.5 & 64.3 \\
\hline Baja & 16.4 & 15.6 & 23.8 & 31.3 & 18.3 \\
\hline
\end{tabular}

Existe una dependencia entre el nivel de conductas conflictivas y el nivel de marginación de la escuela $\left[\mathrm{X}^{2}(6)=17.45, \mathrm{p}=0.008\right]$. Si bien el nivel de conflictividad media es parejo en todos los niveles de marginación, cabe destacar que los estudiantes de escuelas secundarias con bajo nivel de marginación tienen mayor prevalencia de conflictividad alta que el resto de los estudiantes, mientras que los estudiantes de escuelas con muy alto nivel de marginación tienen el valor más alto en nivel de conflictividad bajo en la escala general. Con esto, podemos afirmar que, ante menor presencia de formas de exclusión, es decir, menor nivel de marginación (Conapo, 2016), mayor es la prevalencia de conflictividad alta (ver gráfica 1).

Gráfica 1. Medias de puntajes de conductas conflictivas por nivel de marginación

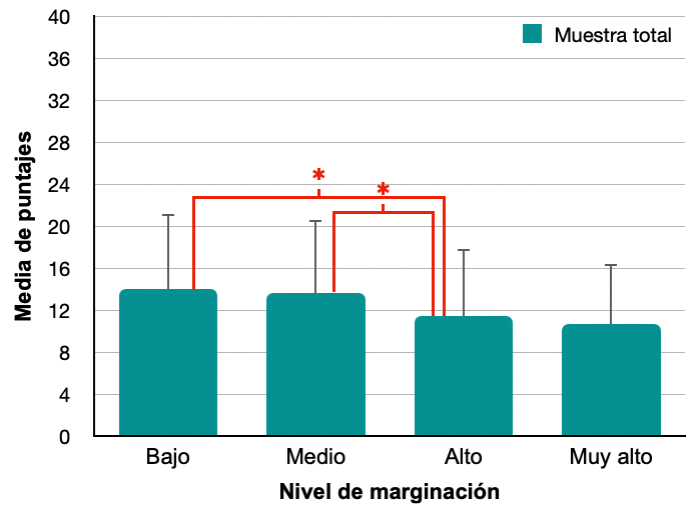

Nota. ${ }^{*} \mathrm{p} \leqq .001$ 
$\mathrm{Al}$ analizar las diferencias de medias de los puntajes de los distintos niveles de marginación mediante ANOVA, obtuvimos $\mathrm{F}(3,645)=5.22$, $\mathrm{p} \leqq 0.001$, de ahí que encontremos diferencias significativas entre los distintos niveles de marginación para los puntajes de conductas conflictivas.

En cuanto al tipo de escuela, la prevalencia de alta conflictividad en secundaria general fue del $23.2 \%$, mientras que en la telesecundaria, del 10.5\%. Los resultados demuestran que existe una dependencia entre el nivel de conductas conflictivas percibidas y el tipo de escuela $\left[X^{2}(2)=26.6, p \leqq 0.001\right]$, y es mayor la prevalencia de conductas conflictivas en las escuelas generales que en las telesecundarias (ver gráfica 2).

Gráfica 2. Medias de puntajes de conductas conflictivas por tipo de escuela

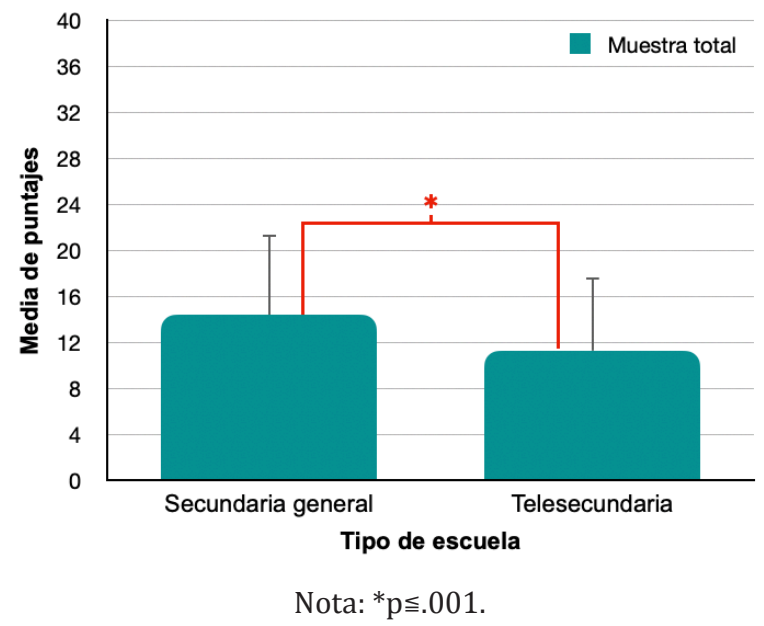

El puntaje medio de la escala general en hombres fue de 13.2 y en mujeres, 12.8; sin embargo, no observamos diferencias significativas por sexo [t(641) $=0.73, \mathrm{p}=0.464]$.

\section{Análisis general de las subescalas}

La subescala de deshonestidad académica percibida en el entorno fue la que obtuvo el mayor puntaje medio, 4.2, con una desviación estándar de 3.2; los puntajes de la subescala de deshonestidad académica vivida mostraron una media de 1.1 y desviación estándar de 1.7; y en la subescala de deshonestidad académica provocada, los datos reportaron una media de 1.2 y desviación estándar de 1.9. Es evidente que hay mayor percepción de las conductas percibidas, en este caso de deshonestidad académica, que de las vividas o provocadas. El puntaje medio de la subescala de desmotivación fue de 3.9, con una desviación estándar de 2.3; este puntaje fue menor que el de deshonestidad percibida en el entorno, pero mayor que el resto de las subescalas. En la escala de disrupción, la media fue de 2.6 y la desviación, de 2.1, lo que la ubica por encima de deshonestidad académica provocada y vivida. 
Análisis de las subescalas por sexo

Con la intención de conocer las diferencias por sexo en cada subescala de conductas conflictivas, realizamos pruebas de diferencia de medias y encontramos diferencias significativas en la subescala de deshonestidad académica percibida en el entorno [t(641)=-2.03, $\mathrm{p}=0.043]$; las mujeres perciben mayor deshonestidad académica en el entorno (4.4) que los hombres (3.9).

En la subescala de deshonestidad académica vivida no distinguimos diferencias entre hombres (1) y mujeres (1.2) [t(641)=-1.64, $\mathrm{p}=0.091]$. Tampoco, diferencias significativas entre hombres (1.2) y mujeres (1.2) en la subescala de deshonestidad académica provocada [t(641) $=-0.15, \mathrm{p}=0.883]$.

En la subescala de desmotivación sí observamos diferencias significativas $[\mathrm{t}(641)=2.3, \mathrm{p}=0.021]$, y los hombres presentan mayores niveles de desmotivación (4.1) que las mujeres (3.7). Del mismo modo, en la subescala de disrupción hay diferencias significativas $[\mathrm{t}(641)=4.56 \mathrm{p} \leqq 0.001]$ y también los hombres (3.1) obtuvieron mayores puntajes que las mujeres (2.3).

Tabla 3. Estadísticos y prevalencia de subescalas de conductas conflictivas

\begin{tabular}{|c|c|c|c|c|}
\hline \multirow{2}{*}{ Subescala } & Sexo & $\begin{array}{c}\text { Media } \pm \text { desv. } \\
\text { estándar }\end{array}$ & Punto de corte & Prevalencia \\
\hline Deshonestidad & & & & \\
\hline \multirow{3}{*}{ Percibida en el entorno } & & & 7.01 & $13.4 \%$ \\
\cline { 2 - 5 } & Hombres & $3.9 \pm 3.1$ & 7.66 & $21.1 \%$ \\
\cline { 2 - 5 } & Mujeres* & $4.4 \pm 3.2$ & 2.7 & $14.5 \%$ \\
\hline \multirow{3}{*}{ Vivida } & Hombres & $1 \pm 1.7$ & 3 & $18.4 \%$ \\
\cline { 2 - 5 } & Mujeres & $1.2 \pm 1.8$ & 3.2 & $10 \%$ \\
\hline \multirow{3}{*}{ Provocada } & & $1.2 \pm 2$ & 3 & $18.4 \%$ \\
\cline { 2 - 5 } & Hombres & $1.2 \pm 1.8$ & & $14.5 \%$ \\
\cline { 2 - 5 } & Mujeres & & 6.5 & $20.1 \%$ \\
\hline \multirow{3}{*}{ Desmotivación } & Hombres* & $4.1 \pm 2.4$ & 5.9 & $14.9 \%$ \\
\cline { 2 - 5 } & Mujeres & $3.7 \pm 2.2$ & 5.2 & $14.2 \%$ \\
\hline \multirow{2}{*}{ Disrupción } & & & 4.3 & \\
\cline { 2 - 5 } & Hombres* & $3.1 \pm 2.2$ & & \\
\cline { 2 - 5 } & Mujeres & $2.3 \pm 2$ & & \\
\hline
\end{tabular}

*Las diferencias son significativas.

La tabla 3 presenta el punto de corte para altos niveles de cada subescala en hombres y mujeres.

Puntuación media para cada subescala de conductas conflictivas por nivel de marginación por sexo

Con la intención de conocer si el nivel de marginación de las escuelas tiene relación con la percepción de conductas conflictivas de los estudiantes, analizamos las relaciones entre cada subescala y la variable nivel de marginación.

La subescala de deshonestidad académica percibida en el entorno es la única en la que identificamos diferencias significativas entre los distintos niveles de marginación 
por sexo; específicamente, en el caso de los hombres encontramos mayores puntajes en los niveles bajo y medio de marginación $[\mathrm{t}(102)=3.29, \mathrm{p}=0.001][\mathrm{t}(235)=2.93$, $\mathrm{p}=0.004]$ que en el alto nivel de marginación. En el caso de las mujeres, también hay diferencias al presentar mayores puntuaciones en el nivel de marginación medio que en el alto $[\mathrm{t}(321)=3.71, \mathrm{p} \leqq 0.001]$. Esto permite leer, de nuevo, que, ante menor presencia de formas de exclusión, mayor es la percepción de deshonestidad académica (ver gráfica 3).
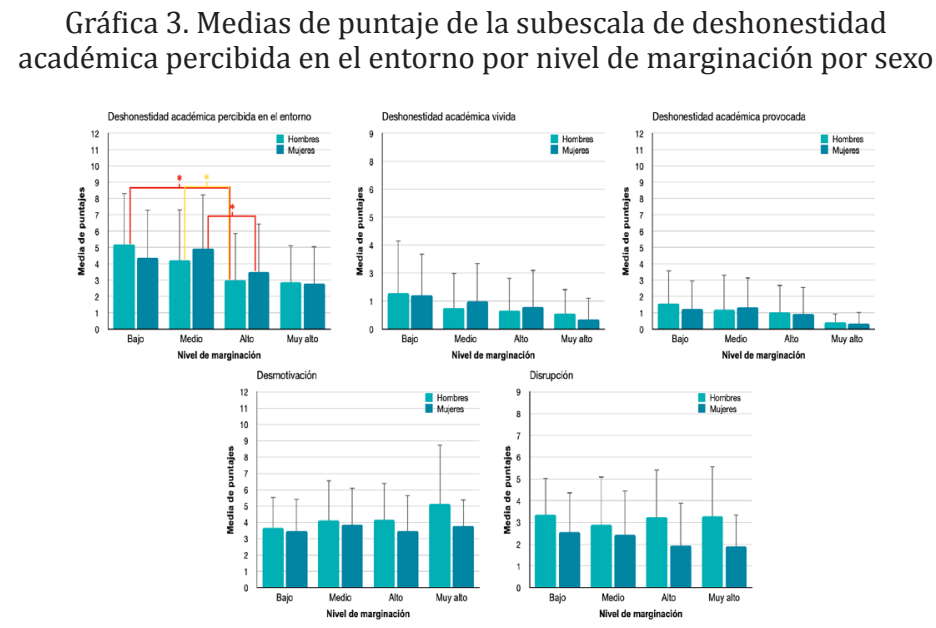

Nota: ${ }^{*} \mathrm{p}<.01,{ }^{*} \mathrm{p} \leqq .001$

Respecto a las demás subescalas (deshonestidad académica vivida, deshonestidad académica provocada, desmotivación y disrupción) no advertimos diferencias significativas entre los distintos niveles de marginación por sexo.

Puntuación media para cada subescala de conductas conflictivas por tipo de escuela por sexo

Del mismo modo, con el nivel de marginación, buscamos conocer si el tipo de escuela (general o telesecundaria) presenta relación con la presencia de conductas conflictivas, por lo que examinamos las relaciones entre cada subescala y la variable nivel tipo de escuela por sexo. La puntuación media se muestra en la tabla 4.

Tabla 4. Puntación media por tipo de escuela y sexo

\begin{tabular}{|c|c|c|c|c|c|c|c|c|}
\hline & \multicolumn{4}{|c|}{ Secundaria general } & \multicolumn{4}{c|}{ Telesecundaria } \\
\cline { 2 - 9 } Conductas conflictivas & $\begin{array}{c}\text { Hom- } \\
\text { bres }\end{array}$ & $\begin{array}{c}\text { Mu- } \\
\text { jeres }\end{array}$ & $\begin{array}{c}\text { Desv. } \\
\text { estándar } \\
\text { hombres }\end{array}$ & $\begin{array}{c}\text { Desv. } \\
\text { estándar } \\
\text { mujeres }\end{array}$ & $\begin{array}{c}\text { Hom- } \\
\text { bres }\end{array}$ & $\begin{array}{c}\text { Muje- } \\
\text { res }\end{array}$ & $\begin{array}{c}\text { Desv. } \\
\text { estándar } \\
\text { hombres }\end{array}$ & $\begin{array}{c}\text { Desv. } \\
\text { estándar } \\
\text { mujeres }\end{array}$ \\
\hline $\begin{array}{c}\text { Deshonestidad académica } \\
\text { percibida en el entorno }\end{array}$ & 4.8 & 5.1 & 3.2 & 3.3 & 3.0 & 3.5 & 2.7 & 2.9 \\
\hline $\begin{array}{c}\text { Deshonestidad académica } \\
\text { vivida }\end{array}$ & 1.1 & 1.4 & 1.9 & 1.9 & 0.8 & 0.9 & 1.5 & 1.6 \\
\hline $\begin{array}{c}\text { Deshonestidad académica } \\
\text { provocada }\end{array}$ & 1.3 & 1.3 & 2.2 & 1.7 & 1.0 & 1.1 & 1.7 & 1.9 \\
\hline Desmotivación & 4.3 & 3.9 & 2.4 & 2.2 & 3.0 & 3.5 & 2.4 & 2.2 \\
\hline Disrupción & 3.0 & 2.6 & 2.2 & 1.9 & 3.1 & 1.9 & 2.1 & 2.0 \\
\hline
\end{tabular}


Del análisis de los puntajes medios por tipo de escuela y sexo, encontramos diferencias significativas para la subescala de deshonestidad académica percibida, ya que, tanto para los hombres como para las mujeres, existe mayor percepción de deshonestidad en el entorno en secundarias generales que en telesecundarias (ver gráfica 6). A partir de estos datos, cabe considerar que las diferencias en la organización de las actividades escolares, el tamaño de la institución en cantidad de estudiantes y docentes, y la ubicación geográfica que existe entre estos dos tipos de escuela pueden estar asociadas al nivel de conflictividad escolar.

Para la subescala de deshonestidad académica vivida, no advertimos diferencias importantes en el caso de los hombres [t(267) $=1.53, \mathrm{p}=0.127]$ por tipo de escuela, pero sí con las mujeres $[\mathrm{t}(372)=2.8, \mathrm{p}=0.005]$; fueron las estudiantes de secundarias generales quienes han vivido mayor deshonestidad académica (ver gráfica 6), lo que significa que han sufrido actos como el que les copien sus tareas o exámenes.

Para las subescalas de deshonestidad académica provocada y desmotivación, no observamos diferencias significativas en los puntajes de tipo de escuela y sexo.

Para la subescala de disrupción, no hubo diferencias significativas en los puntajes de tipo de escuela en el caso de los hombres [t(267) $=-0.29, \mathrm{p}=0.772$ ]; sin embargo, con las mujeres se dieron diferencias significativas $[\mathrm{t}(372)=2.95, \mathrm{p}=0.003]$ y fueron las estudiantes de secundarias generales quienes obtuvieron mayores puntajes de disrupción (ver gráfica 4) respecto de sus pares de telesecundarias.

Gráfica 4. Medias de puntajes de subescalas de conductas conflictivas por tipo de escuela por sexo con diferencias significativas
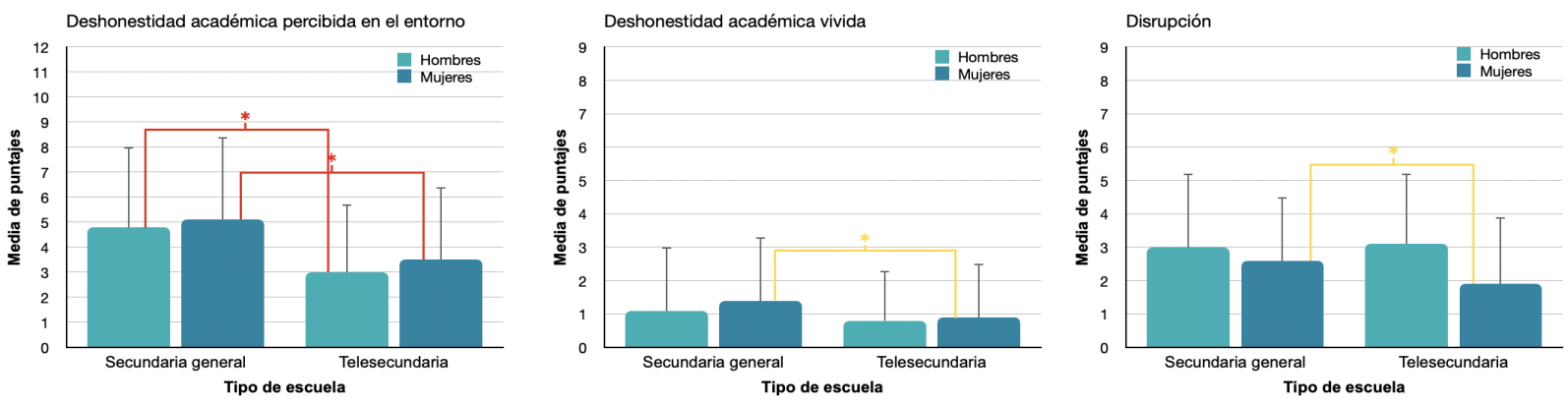

Nota: ${ }^{*} \mathrm{p}<.01,{ }^{*} \mathrm{p}<.001$

Prevalencia de niveles altos de conductas conflictivas por nivel de marginación, tipo de escuela y por sexo

Con la intención de adentrarnos en la problemática de las conductas conflictivas, indagamos las relaciones entre la prevalencia del nivel alto de estas conductas y las variables macro- y meso-, como nivel de marginación y tipo de escuela, y analizamos la asociación entre estas variables.

En primer lugar, cabe mencionar que, por nivel de marginación, la prevalencia de alta conflictividad en la escala global fue del 36\% en el nivel de marginación bajo, $17.1 \%$ en el medio, $12.7 \%$ en el alto y $14.3 \%$ en el muy alto. Observamos asociación 
entre la prevalencia de alta conflictividad y el nivel de marginación $\left[\mathrm{X}^{2}(3)=13.15\right.$, $\mathrm{p}=0.004]$. Esto da cuenta de que, ante menor nivel de marginación, mayor es el puntaje de alta conflictividad.

Por tipo de escuela, la prevalencia de alta conflictividad fue del $23.2 \%$ en secundaria general y del $10.5 \%$ en telesecundaria; existe una asociación entre ambas variables $\left[\mathrm{X}^{2}(1)=17.92, \mathrm{p} \leqq 0.001\right]$, que da cuenta de que, efectivamente, las escuelas generales están asociadas a un mayor nivel de conflictividad.

\section{DiSCUSIÓN Y CONCLUSIONES}

Como mencionamos en la introducción de este trabajo, estudiar el constructo convivencia escolar es teórica y metodológicamente complejo. En este trabajo optamos por abordar una parte de lo que se denomina clima de convivencia, específicamente la percepción de ciertas conductas conflictivas, para relacionarlas con aspectos del mesosistema y macrosistema que pueden determinar, no solo el clima de convivencia, sino la convivencia misma. Los resultados demuestran que las variables consideradas para entender la emergencia de ciertos conflictos adquieren una enorme relevancia; es el caso del nivel de marginación y tipo de escuela y la percepción de conflictos.

De manera global, advertimos una dependencia entre la percepción de conductas conflictivas y el nivel de marginación de la escuela, además de que las escuelas con menores niveles de marginación tuvieron valores más altos de conflictividad, lo cual nos indica que la presencia, o no, de factores de exclusión considerados en los índices de marginación no pueden explicar la incidencia de conflictos en la escuela. En otras palabras, no existen mayores niveles de conflictividad en las escuelas con mayores niveles de marginación. Por otra parte, existe mayor prevalencia de conductas conflictivas en las escuelas generales que en las telesecundarias. Estos resultados contrastan con los presentados por Ascorra et al. (2016, 2019), que muestran que los peores ambientes escolares se observaron en las escuelas municipales de niveles socioeconómicos bajos y no selectivos.

Lo anterior nos lleva a pensar la influencia de otras condiciones del contexto, como la participación en la comunidad (Crespo-Ramos et al., 2017; Varela, Ávila y Martínez, 2013), la organización de la institución, las prácticas docentes y el manejo del currículo respecto a los conflictos que surgen en la cotidianidad escolar (Ochoa y Peiró, 2010; Ochoa y Pérez, 2019; Ochoa, Salinas y Diez-Martínez, 2013). Esto lo podemos explicar desde el modelo ecológico descrito en la introducción de este trabajo y la importancia que tiene para interpretar el cómo se desarrollan las relaciones interpersonales en la escuela (Ochoa y Diez-Martínez, 2013; Ochoa, Diez-Martínez y Garbus, 2020).

En las telesecundarias es un maestro quien imparte todas las materias; suele haber pocos alumnos por salón y provienen de localidades rurales cercanas a la institución escolar (de niveles de marginación medio, alto y muy alto) donde la mayoría se conoce, lo cual puede facilitar la cercanía, así como el afrontamiento y la resolución de los conflictos; además, estas escuelas, por lo regular, mantienen las puertas abiertas a la comunidad. Esto es lo que las distingue de las secundarias generales que están asentadas en localidades urbanas (de nivel de marginación medio y bajo) donde hay distintos profesores para cada materia, que pasan de un salón a otro 
durante su jornada atendiendo a una gran cantidad de estudiantes que provienen de los diversos barrios de la cabecera municipal, así como de algunas localidades aledañas, lo que dificulta el acercamiento con el alumnado.

Por otra parte, es importante apuntar que, del total de la población de estudiantes de secundaria de este municipio, el 54.6\% asiste a las cinco secundarias generales que existen y el otro $45.6 \%$ a las 24 telesecundarias distribuidas en el municipio, lo cual, de entrada, nos arroja un dato relevante, puesto que es evidente que el número de alumnos que se atiende por escuela es un factor determinante para afrontar y resolver los conflictos que surgen en la convivencia escolar cotidiana. En este sentido, habría que indagar, a través de estudios cualitativos, las prácticas específicas que realizan como institución las escuelas telesecundarias que, en este caso, son en las que el estudiantado percibe menor porcentaje de conflictos escolares.

Otro dato relevante que aporta este estudio es que, en general, las mujeres participantes perciben mayores porcentajes de deshonestidad académica en el entorno. De hecho, indagando por tipo de escuela, las mujeres de las secundarias generales son las que la observan en mayor medida. La importancia de estudiar la deshonestidad académica radica en el impacto negativo que esta tiene en la convivencia escolar. Las conductas de deshonestidad académica se aprenden o refuerzan en la escuela y, más aún, la naturalización de estas atenta contra la formación ciudadana, la cual favorece la erradicación de "prácticas que lesionan la vida democrática y el Estado de derecho como la corrupción, el autoritarismo, el nepotismo, la violación a los derechos humanos y la dignidad" (INEE, 2019).

De hecho, la educación para el siglo XXI considera a la escuela como un espacio privilegiado para la enseñanza de la ciudadanía y la democracia (García y De Alba, 2007, citados en Pérez y Ochoa, 2017), por lo que este tipo de conductas deshonestas, en tanto incumplen las normas institucionales, reglamentos de estudiantes, entre otros, afectan tanto la formación en valores como la convivencia democrática.

Llama la atención que sean las mujeres quienes perciben la deshonestidad académica en mayor medida que los hombres. La deshonestidad académica es un constructo que hace referencia a una trasgresión moral. Los estudios pioneros sobre el desarrollo moral fueron realizados por Piaget (1932) y, posteriormente, por Kohlberg (1989), quien considera el desarrollo moral como universal, sin hacer diferencias contextuales y de sexo, lo que suscitó diversas críticas. Una de ellas fue de Guilligan (1982), que mostró que el desarrollo moral evoluciona de modo diferente entre hombres y mujeres debido a las formas de socialización diferenciadas. La autora afirma que las mujeres tienden a ser más cuidadosas y preocupadas por los demás, lo cual puede ser una explicación para este resultado.

Por otro lado, respecto a la deshonestidad académica, es pertinente señalar que estas conductas, en muchas ocasiones, son "provocadas" por las características propias de la escuela, ya que, en general, el dispositivo escolar fomenta el individualismo y la competencia, y para cumplir algunos de estos estándares, promueve acciones de esta naturaleza. Habría que profundizar en el estudio sobre el tipo de prácticas en relación con la deshonestidad académica que se llevan a cabo en las secundarias generales, que son las instituciones en las que las mujeres perciben más la deshonestidad académica (Ochoa y Diez-Martínez, 2012a; Diez-Martínez et al., 2013). 
Por otra parte, los hombres obtuvieron mayores puntajes en relación con la desmotivación y disrupción, lo cual lo podemos explicar a partir de los estereotipos de género. Para Cerro y Vives (2019), los estereotipos de género fungen como mitos que hacen a niñas y niños proclives de violencia $\mathrm{y}$, puesto que los estereotipos se construyen en edades tempranas, en este caso pareciera que los hombres naturalizan la disrupción como un comportamiento "normal" de su género. Coincidimos con Romero y Cardeña (2017) en que los estereotipos de género pueden ser un antecedente de los problemas de convivencia escolar.

Vale la pena destacar el papel de los estereotipos de género como mediadores en las relaciones en el entorno escolar, lo que condiciona también problemas en las interacciones; así, creemos que este es un aspecto fundamental en el cual se debe poner atención para mejorar las relaciones dentro del contexto escolar. Sin embargo, es preciso considerar la dimensión institucional de la convivencia escolar para comprender, por ejemplo, cómo es que las variables indagadas en este estudio muestran condiciones más desfavorables en las secundarias generales que en las telesecundarias, tanto que las mujeres que, en líneas generales, tienen valores más bajos en disrupción y desmotivación que los hombres, cuando se encuentran en contextos como los que caracterizan a las escuelas secundarias generales, estas obtienen valores significativamente más altos que las mujeres de telesecundaria. Sería importante indagar la construcción y función de los estereotipos de género en el ambiente escolar desde una perspectiva ecológica en futuros estudios.

Ahora bien, el que los hombres presenten mayor puntaje medio en la subescala de desmotivación respecto de las mujeres puede explicar que también sean los que registran mayor puntaje medio en la escala de disrupción. En estudios anteriores se demostró que un alto porcentaje del alumnado (97\%) percibía desmotivación (Ochoa y Diez-Martínez, 2012a) y, recientemente, Jurado, Lafuente y Justiniano (2020) mostraron que el ítem que tuvo mayor porcentaje en su estudio fue "te aburres en clase", que dio lugar a explicar la emergencia de la disrupción. Lo anterior nos conduce de nuevo a llamar la atención sobre la necesidad de contextualizar los conflictos en la institución en donde emergen.

Estos resultados muestran la pertinencia del modelo ecológico para el estudio de la convivencia, pues, como pudimos observar, las dimensiones y los subsistemas están en interacción; de esta manera, lo que acontece en cada uno afecta positiva o negativamente en las relaciones de convivencia. La relación demostrada entre nivel de marginación y tipo de escuela, incluso sexo y las variables estudiadas, refleja lo que se sostiene en el modelo ecológico: lo que pasa en la escuela, en este caso conductas conflictivas, no está aislado del contexto en el que ocurre.

Una de las aportaciones principales de nuestra investigación es la relación encontrada entre la percepción de las conductas conflictivas analizadas y el nivel de marginación, pues escasos estudios abordan esta dimensión institucional de la convivencia escolar y, en particular, es poco estudiada en lo relacionado con el macrosistema, que, en este caso, tuvo que ver con el nivel de marginación en el cual se encuentran las escuelas. Para Bronfrenbrenner, el macrosistema es el "más estable y su influencia en los otros sistemas es crítica” (Hernández, 1996, p. 101). El estudio muestra que las variables del macrosistema, en este caso el nivel de marginación, guardan dependencia con la percepción de conductas conflictivas; sin embargo, el 
que sean las escuelas con menos niveles de marginación las que presentan valores más altos de conductas conflictivas demuestra que la vulnerabilidad social no parece desencadenar mayor cantidad de conflictos en el ámbito escolar.

Las comunidades que resultan marginadas presentan niveles elevados de vulnerabilidad social, la cual se determina por las "posibilidades de cumplir un conjunto de condiciones sociales, económicas y espaciales (umbrales) que se asocian al bienestar" (Ruiz, 2012, p. 64). Esta vulnerabilidad no debe abordarse tan solo con acciones individuales, sino que debe convocar políticas y programas que tiendan a cambiar tanto el modelo productivo como los indicadores de bienestar social, de tal forma que todos puedan contar con las mismas oportunidades mediante programas específicos que involucren a la comunidad.

Aquí la escuela tiene una función en particular relevante en la mitigación de los efectos de la marginación en estos grupos sociales. Resulta interesante conocer que, a diferencia de lo que otros estudios han demostrado, en esta investigación las comunidades con mayores niveles de marginación no han sido las que presentaron mayor prevalencia de los conflictos indagados en el ámbito escolar. Este resultado, mencionamos, abre importantes líneas de indagación sobre los alcances de las condiciones macrosociales de la institución y la comunidad y su vínculo con la convivencia escolar.

\section{REFERENCIAS BIBLIOGRÁFICAS}

Aguilera, A., Muñoz, G. y Orozco, A. (2007). Disciplina, violencia y consumo de sustancias nocivas en escuelas primarias y secundarias de México. México: INNE.

Ascorra, P., López, V., Núñez, C., Bilbao, M. A, Gómez, G. y Morales, M. (2016). Relación entre segregación y convivencia escolar en escuela públicas chilenas. Universitas Psychologica, vol. 1, núm. 15, pp. 65-78.

Ascorra, P., López, V. y Urbina, C. (2016). Participación estudiantil en escuelas con buena y mala convivencia escolar. Revista de Psicología, vol. 2, núm. 25, pp. 1-18. https://doi.org/10.5354/0719-0581.2017.44686

Ascorra, P., Ortiz, S., López, V., Núñez, C., Carrasco, C. y Bilbao, M. (2019). Gestión de la convivencia escolar en escuelas vulnerables con altos resultados PISA: convivencia disciplinaria vs. convivencia democrática. En P. Ascorra y V. López (eds.). Una década de investigación en convivencia escolar (pp. 139-160). Chile: Ediciones Universitarias de Valparaíso.

Askell-Williams, H., Cefai, C. \& Fabri, F. (2013). Maltese students' perspectives about their experiences at school and their mental health. Australian Journal of Guidance and Counselling, vol. 2, núm. 23, pp. 252-270. https://doi. org/10.1017/jgc.2013.13

Bronfenbrenner, U. (1987). La ecología del desarrollo humano: cognición y desarrollo humano. Barcelona: Paidós.

Castro, W. O. (2019). La complejidad paradigmática en el aprendizaje significativo de las matemáticas. Revista EDUCARE-UPEL-IPB, segunda nueva etapa 2.0, vol. 23, núm. 2, pp. 77-91.

Cerro, G. M. y Vives, B. M. (2019). Prevalencia de los mitos del amor romántico en jóvenes. OBETS. Revista de Ciencias Sociales, vol. 2, núm. 14, pp. 343-371. https://doi.org/10.14198/OBETS2019.14.2.03 
Chen, J. K. \& Wei, H. S. (2011). the impact of school violence on self-esteem and depression among Taiwanese Junior High School students. Social Indicators Research, vol. 100, núm. 3, pp. 479-498. https://doi.org/10.1007/s11205010- 9625-4

Conapo (2016). Índice de Marginación por Entidad Federativa y Municipio 2015. México. https://www.gob.mx/conapo/documentos/indice-de-marginacion-por-entidad-federativa-y-municipio-2015

Crespo-Ramos, S., Romero-Abrio, A., Martínez-Ferrer, B. y Musitu, G. (2017). Variables psicosociales y violencia escolar en la adolescencia. Psychosocial Intervention, vol. 2, núm. 26, pp.125-130. https://doi.org/10.1016/j. psi.2017.05.002

Coordinación General @prende.mx (2020). La Telesecundaria celebra su 52 aniversario. https://www.gob.mx/aprendemx/articulos/la-telesecundariacelebra-su-52-aniversario?idiom $=\mathrm{es}$

Diez-Martínez, E., Ochoa, A., Vázquez, F., Gilio, C., Sahagún, G. y Valdivia, C. (2013). Algunos aspectos de la deshonestidad académica en la educación superior: reflexiones acerca de la formación ciudadana. Ponencia presentada en el XII Congreso Nacional de Investigación Educativa, 18 a 22 de noviembre. Guanajuato, Guanajuato.

Eisenberg, J. (2004). To cheat or not to cheat: Effects of moral perspective situational variables on students' attitudes. Journal of Moral Education, vol. 33, núm. 2, pp. 163-178.

Flaspohler, P. D., Elfstrom, J. L., Vanderzee, K. L., Sink, H. E. y Birchmeier, Z. (2009). Stand by me: The effects of peer and teacher support in mitigating the impact of bullying on quality of life. Psychology in the Schools, vol. 7, núm. 46, pp. 636-649. https://doi.org/10.1002/pits.20404

Gaeta, M. L., Martínez-Otero, V., Rodrigo, M. y Rodrigo, G. (2020). Problemas de convivencia escolar desde la mirada del alumnado de educación secundaria. Estudios Pedagógicos, vol. XLVI, núm. 2, pp. 341-357.

Gordillo, E., Rivera-Calcina, R. y Gamero, G. (2014). Conductas disruptivas en escuelas diferenciadas, coeducativas, interactivas. Educ., vol. 3, núm. 17, pp. 427-443. https://doi.org/10.5294/edu.2014.17.3.2

Grau Vidal, R. y García Raga, L. (2017). Prácticas socioeducativas para mejorar la convivencia escolar. Una experiencia en un centro de acción educativa singular (Valencia, España). Revista sobre la Infancia y la Adolescencia, núm. 12, pp. 42-50. https://doi.org/10.4995/reinad.2017.6490

Guilligan, C. (1982). In a different voice: Psychological theory and women's development. Cambridge University Press.

Hernández, C. (1996). Bronfenbrenner y los modelos ecológicos. En R. Clemente y C. Hernández. Contextos de desarrollo psicológico y educación. Málaga: Aljibe.

INEE (2019). Condiciones básicas para el aprendizaje en los preescolares de México. México.

INEE (2018). Panorama educativo de México. Indicadores del sistema educativo nacional 2017. Educación básica y media superior. México.

Jurado, P., Lafuente, A. y Justiniano, D. (2020). Conductas disruptivas en educación secundaria obligatoria: análisis de factores intervinientes. Contextos Educativos, núm. 25, pp. 219-236 https://doi.org/10.18172/con.3827 
Jurado, P. y Tejada, J. (2019). Disrupción y fracaso escolar. Un estudio en el contexto de la educación secundaria obligatoria en Cataluña. Estudios sobre Educación, núm. 36, pp.135-155 https://doi.org/10.15581/004.36.135-155

Kohlberg, L. (1989). Estadios morales y moralización. El enfoque cognitivo-evolutivo. En E. Turiel, I. Enesco y J. Linaza. El mundo social en la mente infantil. Madrid: Alianza.

Martínez, M. B., Chacón, J. C., Díaz-Aguado, M. J., Martín, J. y Martínez, R. (2020). La disrupción en las aulas de ESO: un análisis multi-informante de la percepción de profesores y alumnos. Pulso, núm. 43, pp. 15-34.

Morey-López, M., Sureda-Negre, J., Oliver-Trobat, M. F. y Comas-Forgas, R. L. (2013). Plagio y rendimiento académico entre el alumnado de educación secundaria obligatoria. Estudios sobre Educación, núm. 24, pp. 225-244. https:// dadun.unav.edu/bitstream/10171/29571/2/MOREY.pdf

Murdock, T. B., Hale, N. \& Weber, M. J. (2001). Predictors of cheating among early adolescents: Academic and social motivations. Contemporary Educational Psychology, vol. 3, núm. 4, pp. 129-145.

Nora, W. \& Zhang, K. (2010). Motives of cheating among secondary students: The role of self-efficacy and peer influence. Asia Pacific Educ. Rev., núm. 11, pp. 573-584. https://doi.org/10.1007/s12564-010-9104-2

Ochoa, A. (2019). El tipo de participación que promueve la escuela, una limitante para la inclusión. Alteridad Revista de Educación, vol. 2, núm.14, pp. 184194. https://doi.org/10.17163/alt.v14n2.2019.03

Ochoa, A., Salinas, J. y Diez-Martínez, E. (2013). La desmotivación de estudiantes y la deshonestidad académica: ¿problemas de convivencia? En Memoria del XII Congreso Nacional de Investigación Educativa. Consejo Mexicano de Investigación Educativa, Guanajuato, Guanajuato, 18-22 de noviembre.

Ochoa, A. y Diez-Martínez, E. (2013). El reglamento escolar como eje de análisis de la convivencia en la escuela. Ensaio: aval. pol. púb. Educ., núm. 21, vol. 18, pp. 667-684. http://dx.doi.org/10.1590/S0104-40362013000400003

Ochoa, A. y Diez-Martínez, E. (2012a). ¿Formación cívica y ética? La corrupción como actos cotidianos en la escuela. Ponencia presentada en la IX Jornada Nacional de Investigadores en Educación y Valores, 20 al 22 de septiembre. Guadalajara, Jalisco, México.

Ochoa, A. y Diez-Martínez, E. (2012b). La escuela como sistema social de convivencia y sus implicaciones en algunos problemas de violencia. La percepción de estudiantes de primaria y secundaria. Diálogos sobre Educación, vol. 3, núm. 4. http:// dialogossobreeducacion.cucsh.udg.mx/index.php/DSE/article/view/363

Ochoa, A. y Peiró, S. (2010). Estudio comparativo de las actuaciones de los profesores ante situaciones que alteran la convivencia escolar: el caso de Querétaro (México) y Alicante (España). Revista Electrónica Interuniversitaria de Formación del Profesorado, vol. 4, núm.13. http://www.aufop.com

Ochoa, A. y Pérez, L. (2019). El aprendizaje servicio, una estrategia para impulsar la participación y mejorar la convivencia escolar. Psicoperspectivas. Individuo y Sociedad, vol. 1, núm. 18, pp.1-13. https://doi.org/10.5027/psicoperspectivas-vol18-issue1-fulltext-1478

Ochoa Cervantes, A., Diez-Martínez Day, E. y Garbus, P. (2020). Análisis del concepto de participación en estudiantes de secundarias públicas. Sinéctica, Revista Electrónica de Educación, núm. 52, pp.1-19. https://doi.org/10.31391/ S2007-7033(2020)0054-003 
Ortega, R. y Del Rey, R. (2006). Construir la convivencia. España: Edebé.

Pérez Galván, L. M. y Ochoa Cervantes, A. (2017). La participación de los estudiantes en una escuela secundaria: retos y posibilidades para la formación ciudadana. Revista Mexicana de Investigación Educativa, vol. 22, núm. 72, pp. 179-207. http://www.scielo.org.mx/scielo.php?script=sci_ arttext\&pid=S1405-66662017000100179\&lng=es\&tlng=es

Piaget, J. (1932). El criterio moral en el niño. Barcelona: Fontanella.

Reskala, F. (2020). Nuevos comportamientos de deshonestidad académica en estudiantes mexicanos: un estudio exploratorio. Informes Psicológicos, vol. 20, núm. 2, pp. 155-170.

Romero, M. y Cardeña, C. (2017). Validación psicométrica de un instrumento para medir estereotipos de género en niños de educación primaria. En CONISEN. Memorias del Congreso Nacional de Investigación sobre Educación Normal (pp. 1-14). Mérida, México. https://bit.ly/3uQjbA7

Ruiz Rivera, N. (2012). La definición y medición de la vulnerabilidad social. Un enfoque normativo. Investigaciones Geográficas, núm. 77, pp. 63-74.

Salinas de la Vega, J. y Ochoa Cervantes, A. (2016). Escala para la valoración de conflictos que afectan la convivencia escolar (ECACE). Actas del IV Congreso Internacional en Contextos Psicológicos, Educativos y Salud, núm. 1, p. 86. http://oce.uaq.mx/docs/Investigacion/ConvivenciaEscolar/Salinas_ Ochoa_2016_Escala_para_la_valoracion_de_conflictos_que_afectan_la_convivencia_escolar_ECACE.pdf

Sureda, J., Comas, R. y Oliver, M. (2015). Plagio académico entre estudiantes de secundaria y bachillerato: diferencias en cuanto al género y procastinación. Comunicar Revista Científica de Educomunicación, vol. XXII, núm. 44, pp. 103111. https://doi.org/10.3916/C44-2015-11

Tijmes, C. (2012). Violencia y clima escolar en establecimientos educacionales en contextos de alta vulnerabilidad social de Santiago de Chile. PSYKHE, vol. 2, núm. 21, pp.105-117. http://www.psykhe.cl/index.php/psykhe/article/ view/548

Torres-Rivera, A. y Florencio, R. (2019). Aprender a convivir en educación superior desde la práctica docente para una sociedad democrática. Formación Universitaria, vol. 2, núm. 12, pp. 51-62. https://doi.org/10.4067/S071850062019000200051

Trianes Torres, M. V., Blanca Mena, M. J., Fernández Baena, F. J., Escobar Espejo, M., Maldonado Montero, E. F. y Muñoz Sánchez, Á. (2009). Evaluación del estrés infantil: inventario infantil de estresores cotidianos (IIEC). Psicothema, vol. 4, núm. 21, pp. 598-603. http://www.psicothema.com/pdf/3677.pdf

Varela Garay, R. M., Ávila, M. y Martínez, B. (2013). Violencia escolar: un análisis desde los diferentes contextos de interacción. Psychosocial Intervention, vol. 1, núm. 22, pp. 25-32. https://doi.org/10.50

Villa Romero, A. R., Alvarado León, N. Y., Gayol Pérez, E. y Payán Fierro, M. E. (2012). Introducción a los diseños epidemiológicos. En A. R. Villa Romero, L. Moreno Altamirano y G. S. García de la Torre (eds.). Epidemiología y Estadística en Salud Pública (pp. 62-67). McGraw-Hill Educación. 